\title{
At-wavelength characterization of the EUV Engineering Test Stand Set-2 optic
}

Patrick Naulleau ${ }^{1}$, Kenneth A. Goldberg ${ }^{1}$, Erik H. Anderson ${ }^{1}$, Phillip Batson ${ }^{1}$, Paul E. Denham ${ }^{1}$, Keith H. Jackson ${ }^{1}$, Eric M. Gullikson ${ }^{1}$, Senajith Rekawa ${ }^{1}$, and Jeffrey Bokor ${ }^{1,2}$

${ }^{1}$ Center for X-Ray Optics, Lawrence Berkeley National Laboratory, Berkeley, CA 94720

${ }^{2}$ EECS Department, University of California, Berkeley, CA 94720

At-wavelength interferometric characterization of a new 4x-reduction lithographicquality extreme ultraviolet (EUV) optical system is described. This state-of-the-art projection optic was fabricated for installation in the EUV lithography Engineering Test Stand (ETS) and is referred to as the ETS Set-2 optic. EUV characterization of the Set-2 optic is performed using the EUV phase-shifting point diffraction interferometer (PS/PDI) installed on an undulator beamline at Lawrence Berkeley National Laboratory's Advanced Light Source. This is the same interferometer previously used for the at-wavelength characterization and alignment of the ETS Set-1 optic.

In addition to the PS/PDI-based full-field wavefront characterization, we also present wavefront measurements performed with lateral shearing interferometry, the chromatic dependence of the wavefront error, and the system-level pupil-dependent spectral-bandpass characteristics of the optic; the latter two properties are only measurable using at-wavelength interferometry.

Contact: Patrick Naulleau

LBNL, MS 2-400, 1 Cyclotron Rd., Berkeley, CA 94720

Tel: 510-486-4529 | Fax: 510-486-4550 | Email: Pnaulleau@lbl.gov 


\section{Introduction}

The recent interest in extreme ultraviolet (EUV) lithography [1] has led to the development of a variety of novel metrologies. Because EUV optical systems utilize resonant-stack, reflective multilayer-coated optics [2], performing metrology at the operational wavelength is essential to the development process [3]. This has led to numerous advancements in the field of EUV interferometry [4-10]. With a demonstrated reference-wavefront accuracy of better than $\lambda_{\mathrm{EUV}} / 350\left(0.04 \mathrm{~nm}\right.$ at $\left.\lambda_{\mathrm{EUV}}=13.4 \mathrm{~nm}\right)[\mathbf{1 0}]$, the PS/PDI $[\mathbf{6 , 7}]$ is, to the best of our knowledge, the highest accuracy EUV interferometer available.

Operating at the Advanced Light Source (ALS) synchrotron radiation facility at Lawrence Berkeley National Laboratory (LBNL), the PS/PDI has been in use for several years in the measurement and alignment of numerous small-field EUV 10×-reduction Schwarzschild objectives [11]. Independent verification of the accuracy of the PS/PDI and its utility in predicting and optimizing imaging performance has come from ongoing lithographic exposure experiments conducted at Sandia National Laboratories [12].

More recently, a new interferometry endstation was constructed [8] specifically for atwavelength testing of projection optics designed for the EUV-Lithography Engineering Test Stand (ETS) [13]. This interferometer was used last year to characterize and align [8] the first of two projection optical systems [14] (the ETS Set-1 optic) which is currently installed in the operational ETS. Since that time, a second projection optic of higher quality (the ETS Set-2 optic) has been fabricated and assembled. The projection optics are four-mirror, aspheric ring-

field systems, designed to operate at approximately $13.4-\mathrm{nm}$ wavelength with a numerical aperture (NA) of 0.1 and $4 \times$ demagnification. 
Here we describe the characterization of the ETS Set-2 optic using at-wavelength interferometry. Installed on an undulator beamline, the PS/PDI (Fig. 1) is a versatile metrology tool, which can be configured to perform a variety of system-level interferometric and noninterferometric measurements. The interferometer is readily configurable as a lateral shearing interferometer [9] providing a significant increase in aberration magnitude measurement range, which is useful for initial system alignment. We present the use of the PS/PDI for the full-field wavefront characterization of the ETS Set-2 optic. In addition to field-dependent wavefront measurements, we also present the chromatic dependence of the wavefront error, and the systemlevel pupil-dependent spectral-bandpass characteristics of the optic, properties that are only measurable at-wavelength.

\section{PS/PDI Configuration}

The PS/PDI was constructed to evaluate the system wavefront at arbitrary positions across the field of view. In the object plane, the ring field is arc-shaped, spanning 104-mm by $6 \mathrm{~mm}$ wide, and subtending 29-degrees. Measurements of the field-dependent optical performance, across the large ring-field, provide feedback for the alignment of the individual mirror elements, enabling optimal imaging quality to be achieved.

The PS/PDI design (Fig. 1) has only a few critical optical components. All of the components exist in a vacuum environment with a base pressure of $10^{-7}$ Torr; a partial pressure of $10^{-5}$ Torr of oxygen gas is introduced as a pre-emptive contamination mitigation measure. Great care is taken to follow clean, UHV practices, and to ensure that only UHV compatible materials are introduced into the vacuum chamber. Once the interferometer component alignments were complete, an insulated thermal enclosure was constructed to contain the interferometer endstation and maintain temperature stability of $0.01 \mathrm{C}$ over 8 hours. 
Within the vacuum chamber, a synchrotron beam from an undulator source is focused onto the object (reticle) plane of the test optic from above; the illumination angle and position matches the design conditions. A Kirkpatrick-Baez glancing-incidence optical system focuses the beamline radiation into a fixed spot nominally $5-\mu \mathrm{m}$ wide.

In the object (reticle) plane, diffraction from a small pinhole (the object pinhole) produces spatially coherent, spherical-wave illumination of the test optic, filling the pupil of the optical system with a divergence angle significantly larger than the input numerical aperture (NA) of the system. This guarantees the spherical accuracy of the probe (test) beam. A grating beamsplitter placed between the object pinhole and the test optic creates a series of overlapping coherent beams that are focused to laterally displaced positions in the image (wafer) plane. The imageplane beam separation is designed to be $5 \mu \mathrm{m}$. By propagating through the optical system, each of these overlapping beams acquires the characteristic aberrations of the test optic. In the imageplane a patterned opaque and transparent (open stencil) mask selects two adjacent beams with all other beams being blocked. One of the two beams, the test beam, passes through a relatively large window in the mask, thereby preserving the aberrations imparted by the optical system. The second unblocked beam is focused onto a pinhole (the reference pinhole) smaller than the diffraction-limited resolution of the test optic, thereby producing an ideally spherical reference beam. The two beams propagate to the CCD where they overlap creating an interference pattern that records the deviation of the test beam from an ideal sphere.

To enable characterization of the test optic across the large field of view, the extended field is divided into 45 discrete field points, prescribed by the alignment method. The object and image-plane masks are comprised arrays of pinholes and alignment features arranged to coincide with each field point position. The entire interferometer, including the test optic and the pinhole 
arrays, is moved under the stationary undulator beam, allowing the field points to be measured sequentially.

The pinhole arrays are fabricated with electron beam lithography and reactive ion etching at LBNL's Nanowriter facility [15]. The masks are made up of a 200-nm-thick nickel absorbing layer evaporated onto 100 -nm-thick low-stress silicon-nitride $\left(\mathrm{Si}_{3} \mathrm{~N}_{4}\right)$ membranes. The mask features are etched completely through the membrane prior to the Ni evaporation. Thus the pinholes and windows are completely open in the finished masks, which maximizes their transmission and significantly mitigates contamination problems.

\section{Measurement Results}

Prior to EUV characterization at LBNL, the ETS Set-2 optic was assembled, characterized, and aligned using visible-light interferometry at Lawrence Livermore National Laboratory [16]. The alignment was performed using an alignment algorithm developed for both EUV and visible-light interferometry [17]. The system was then brought to LBNL for at-wavelength characterization and re-alignment if were deemed necessary.

\section{Wavefront measurements}

At LBNL, three complete sets of interferometric measurements were performed over a fiveweek time period. Two initial measurements were performed using the shearing configuration [9] as the system temperature was reduced from the ALS ambient temperature of $24.5 \mathrm{C}$ to the designed operating temperature, 21.0 C. All three measurements where performed at the optic centroid wavelength of $13.55 \mathrm{~nm}$.

The two shearing measurements were performed at $22.6 \mathrm{C}$ and $20.9 \mathrm{C}$, respectively. Although not described here in detail, some minor wavefront temperature dependence $(\sim \lambda / 100$ rms) was observed. Following stabilization of the interferometer endstation temperature, the 
system was configure for PS/PDI measurements and the entire field was again characterized. As described below, good agreement was found between the shearing and PS/PDI measurements.

Good qualitative agreement was also found between the visible-light measurements and EUV measurements and a more rigorous quantitative comparison is underway. The EUV measurements revealed that the system alignment had not changed appreciably during transport, temperature adjustment, and during the five weeks between the visible-light and EUV measurements. Due to the fact that qualitative agreement was found, no subsequent alignment was performed based on the EUV measurements.

Figure 2 shows wavefront-characterization results determined from the final PS/PDI measurement. Because full phase-shifting analysis has not yet been done, these results should be considered as preliminary and small changes in the final rms numbers should be expected. Figure 2 shows the individual wavefronts measured at each of the 45 different field points as well as a contour map of the rms error across the field. The rms wavefront errors listed below each wavefront are in $\mathrm{nm}$ and are based on a 37-term Zernike Polynomial fit to the wavefront [18] with the measurement-dependent piston, tilt, and focus terms removed. The depicted wavefronts include higher spatial frequency content than is contained within the 37-term Zernike polynomial reconstructions. At the best field point, a significant improvement in wavefront quality (a factor of approximately 1.75) has been found relative to the Set-1 optic [8].

Figure 3 shows the comparison between the final shearing measurement and the final PS/PDI measurement. The contour maps are based on the rms error over a NA of 0.0915 as limited by the measurement NA of the shearing implementation that was used [9]. The shearing measurement was performed using $2-\mu \mathrm{m}$ pitch gratings placed approximately $300 \mu \mathrm{m}$ from focus. For the comparison, the PS/PDI data was re-analyzed over the same $30 \times 30$ pixel grid size 
as used for the shearing. The coarser grid for shearing arises from the shear angle magnitude which is $1 / 15$-th of the -NA angle. Between the shearing and PS/PDI, the average agreement across the field, as determined by the rms magnitude of the difference wavefront, was found to be $(0.25 \pm 0.06) \mathrm{nm}(\lambda / 53)$ with the best agreement being $0.12 \mathrm{~nm}(\lambda / 111)$.

\section{Chromatic aberrations}

One of the unique capabilities of the EUV interferometer is its ability to measure chromatic dependence of the wavefront near the designed operating wavelength [19]. In these measurements a single field point is interferometrically probed as the illumination wavelength is tuned through a range exceeding the optic full-width-half-max spectral passband. Figure 4 shows the wavefront change as a function of wavelength relative to the wavefront measured at 13.35 $\mathrm{nm}$ wavelength. This measurement was performed at the central field point where the rms wavefront error is approximately $0.6 \mathrm{~nm}$. With a difference-wavefront precision measured at approximately $0.006 \mathrm{~nm}$ (based on repeated measurements performed at a single wavelength), the results in Fig. 4 indicate the optic to be essentially free of chromatic aberrations.

\section{Spectral bandpass}

In addition to performing wavefront measurements, the PS/PDI is also well suited to the characterization of system-level spectral-bandpass measurements. Moreover, because the optical system pupil is effectively projected onto the $\mathrm{CCD}$, the spectral characteristics can be determined as a function of pupil position. In this case the grating beamsplitter is removed from the system and pupil transmission images are recorded on the CCD as the wavelength is varied. Each CCD pixel is then treated as an independent detector from which the spectral response for the corresponding point in the pupil can be found. 
Figure 5 shows the centroid wavelength change as a function of pupil position as measured at the central field point. The pupil map has been binned down to a 40-pixel grid. The average centroid wavelength is $13.35 \mathrm{~nm}$ with a peak-to-valley linear variation of $(0.015 \pm 0.002) \mathrm{nm}$ across the pupil. The variation, which is oriented along the direction of large angles of incidence, is consistent with that expected from the optical design and the known coating parameters, which were measured after the coating of each individual mirror. Modeling results show an expected linear change of approximately $0.017 \mathrm{~nm}$ across the pupil.

\section{Conclusion}

At-wavelength characterization of the ETS Set-2 optic has been completed and significant improvement of the Set-1 optic [8] was found. This characterization included both shearing and PS/PDI measurements across the field, chromatic aberration measurements, and pupil-positiondependent spectral passband measurements. The spectral and chromatic measurements, which can only be performed at wavelength, demonstrated the extremely high quality of the EUV coatings. Having completed the at-wavelength characterization, the PS/PDI is now undergoing modifications that will allow it to be used to perform static printing experiments across the field [20]. These capabilities will allow the earliest possible imaging demonstration with the Set-2 optic before it is installed into the ETS for full-field scanned imaging.

\section{Acknowledgements}

The authors are greatly indebted to Bruce Harteneck, Deirdre Olynick, and Eugene Veklerov for nanofabrication support, to Farhad Salmassi for coating support, to David Richardson and Ron Tackaberry for programming support, and to Brian Hoef, Drew Kemp, Gideon Jones, and Rene Delano for technical and fabrication support. This research was supported by the Extreme Ultraviolet Limited Liability Company and the DOE Office of Basic Energy Science. 


\section{References}

1. R. Stulen and D. Sweeney, "Extreme ultraviolet lithography," IEEE J. Quantum Electron. 35, 694-699 (1999).

2. J. H. Underwood and T. W. Barbee, Jr., "Layered synthetic microstructures as Bragg diffractors for X rays and extreme ultraviolet: theory and predicted performance,” Appl. Opt. 20, 3027-3034 (1981).

3. D. Attwood, G. Sommargren, R. Beguiristain, K. Nguyen, J. Bokor, N. Ceglio, K. Jackson, M. Koike, and J. Underwood, "Undulator radiation for at-wavelength interferometry of optics for extreme-ultraviolet lithography,” Appl. Opt. 32, 7022-7031 (1993).

4. J. E. Bjorkholm, A. A. MacDowell, O. R. Wood II, Z. Tan, B. LaFontaine, and D. M. Tennant, "Phase-measuring interferometry using extreme ultraviolet radiation,” J. Vac. Sci. \& Technol. B 13, 2919-2922 (1995).

5. A. K. Ray-Chaudhuri, K. D. Krenz, and C. H. Fields, "At-wavelength characterization of an extreme ultraviolet camera from low to mid-spatial frequencies with a compact laser plasma source," J. Vac. Sci. Technol. B 15, 2462-2466 (1997).

6. H. Medecki, E. Tejnil, K. A. Goldberg, and J. Bokor, "Phase-shifting point diffraction interferometer," Opt. Lett. 21, 1526-1528 (1996).

7. K. A. Goldberg, "Extreme Ultraviolet Interferometry," Ph.D. dissertation (University of California, Berkeley, 1997).

8. K. A. Goldberg, P. Naulleau, P. Batson, P. Denham, E. Anderson, H. Chapman, and J. Bokor, "EUV alignment and testing of a four-mirror ring field EUV optical system," J. Vac. Sci. \& Technol. B 18, 2911-2915 (2000). 
9. P. Naulleau, K. A. Goldberg, and J. Bokor, "EUV carrier-frequency shearing interferometry of a lithographic four-mirror optical system,” J. Vac. Sci. \& Technol. B 18, 2939-2943 (2000).

10. P. Naulleau, K. Goldberg, S. Lee, C. Chang, D. Attwood, and J. Bokor, "The EUV phaseshifting point diffraction interferometer: a sub-angstrom reference-wave accuracy wave front metrology tool," Appl. Opt. 38, 7252-7263 (1999).

11. K. A. Goldberg, P. Naulleau, and J. Bokor, "EUV interferometric measurements of diffraction-limited optics,” J. Vac. Sci. \& Technol. B 17, 2982-2986 (1999).

12. G. F. Cardinale, C. C. Henderson, J. E. M. Goldsmith, P. J. S. Mangat, J. Cobb, and S. D. Hector, "Demonstration of pattern transfer into sub-100 nm polysilicon line/space features patterned with extreme ultraviolet lithography,” J. Vac. Sci. \& Technol. B 17, 2970-2974 (1999).

13. D. Tichenor, A. Ray-Chaudhuri, W. Replogle, R. Stulen, G. Kubiak, P. Rockett, L. Klebanoff, K. Jefferson, A. Leung, J. Wronosky, L. Hale, H. Chapman, J. Taylor, J. Folta, C. Montcalm, R. Soufli, E. Spiller, K. Blaedel, G. Sommargren, D. Sweeney, P. Naulleau, K. Goldberg, E. Gullikson, J. Bokor, P. Batson, D. Attwood, K. Jackson, S. Hector, C. Gwyn, and P. Yan , "System Integration and Performance of the EUV Engineering Test Stand," Proceedings of the SPIE Vol. 4343, to be published (2001).

14. D. W. Sweeney, R. Hudyma, H. N. Chapman, and D. Shafer, "EUV optical design for a 100 nm CD imaging system," in Emerging Lithographic Technologies II, Y. Vladimirsky, ed., Proc. SPIE Vol. 3331, 2-10 (1998).

15. E. H. Anderson, V. Boegli, and L. P. Murray, "Electron beam lithography digital pattern generator and electronics for generalized curvilinear structures J. Vac. Sci. \& Technol. B 13, 2529-2534 (1995). 
16. G. E. Sommargren, "Phase shifting diffraction interferometry for measuring extreme ultraviolet optics," OSA Trends in Optics and Photonics Vol. 4, Extreme Ultraviolet Lithography, G.D. Kubiak and D.R. Kania, eds. (Optical Society of America, Washington, DC 1996), pp. 108-112.

17. H. N. Chapman and D. W. Sweeney, "A rigorous method for compensation selection and alignment of microlithographic optical systems," Proceedings of the SPIE Vol. 3331, 102-113 (1998).

18. A. Bathia and E. Wolf, "The Zernike circle polynomials occurring in diffraction theory," Proc. Phys. Soc. B65, 909-910 (1952).

19. E. Tenjil, K. A. Goldberg, and J. Bokor, "Phase effects owing to multilayer coatings in a twomirror extreme-ultraviolet Schwarzschild objective,” Appl. Opt. 37, 8021-8029 (1998).

20. P. Naulleau, K. Goldberg, E. Anderson, P. Batson, P. Denham, S. Rekawa, and J. Bokor, "Adding static printing capabilities to the EUV phase-shifting point diffraction interferometer," Proceedings of the SPIE Vol. 4343, to be published (2001). 


\section{List of Figures}

Fig. 1. Schematic of the EUV PS/PDI installed at an undulator beamline at Lawrence Berkeley National Laboratory's Advanced Light Source synchrotron radiation facility. A KirkpatrickBaez glancing-incidence optical system focuses the beamline radiation into a nominally-5- $\mu \mathrm{m}$ spot in the test optic object plane. Pinhole diffraction is used to produce both the probe and reference waves and a transmission grating is used as the beamsplitter.

Fig 2 Wavefronts measured at each of the 45 different field points and contour map of the rms error across the field. The rms wavefront errors listed below each wavefront are in $\mathrm{nm}$ and are based on a 37-term Zernike Polynomial fit to the wavefront with the measurement-dependent piston, tilt, and focus terms removed. The depicted wavefronts include higher spatial frequency content than is contained within the 37-term Zernike polynomial reconstructions. Each wavefront image is individually scaled.

Fig. 3. Comparison between the final shearing measurement and the final PS/PDI measurement (both at-wavelenth). The contour maps are based on the rms error over a numerical aperture (NA) of 0.0915 as limited by the measurement NA of the shearing implementation used. For the comparison, the PS/PDI data was re-analyzed over the same grid size and NA as used for the shearing. The average agreement across the field is $(0.25 \pm 0.06) \mathrm{nm}$.

Fig. 4. Wavefront variation as a function of wavelength relative to the wavefront measured at a wavelength of $13.35 \mathrm{~nm}$. This measurement was performed at the central field point where the wavefront error is approximately $0.6 \mathrm{~nm} \mathrm{rms}$.

Fig. 5. Centroid wavelength change as a function of pupil position as measured at the central field point. The average centroid wavelength is $13.35 \mathrm{~nm}$ with a peak-to-valley linear variation of 
$(0.015 \pm 0.002) \mathrm{nm}$ across the pupil. Modeling results show an expected linear change of approximately $0.017 \mathrm{~nm}$ across the pupil. 
Printed at full width of 19picas, do not reduce.

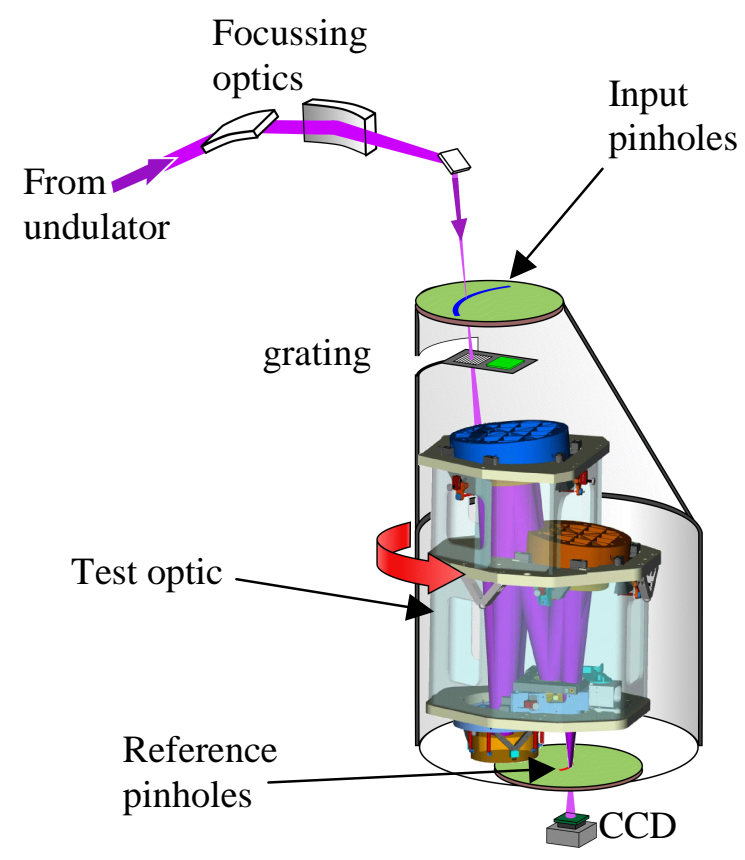

Fig. 1. Schematic of the EUV PS/PDI installed at an undulator beamline at Lawrence Berkeley National Laboratory's Advanced Light Source synchrotron radiation facility. A Kirkpatrick-Baez glancing-incidence optical system focuses the beamline radiation into a nominally-5- $\mu \mathrm{m}$ spot in the test optic object plane. Pinhole diffraction is used to produce both the probe and reference waves and a transmission grating is used as the beamsplitter. 
Printed at full width of 35 picas, do not reduce.

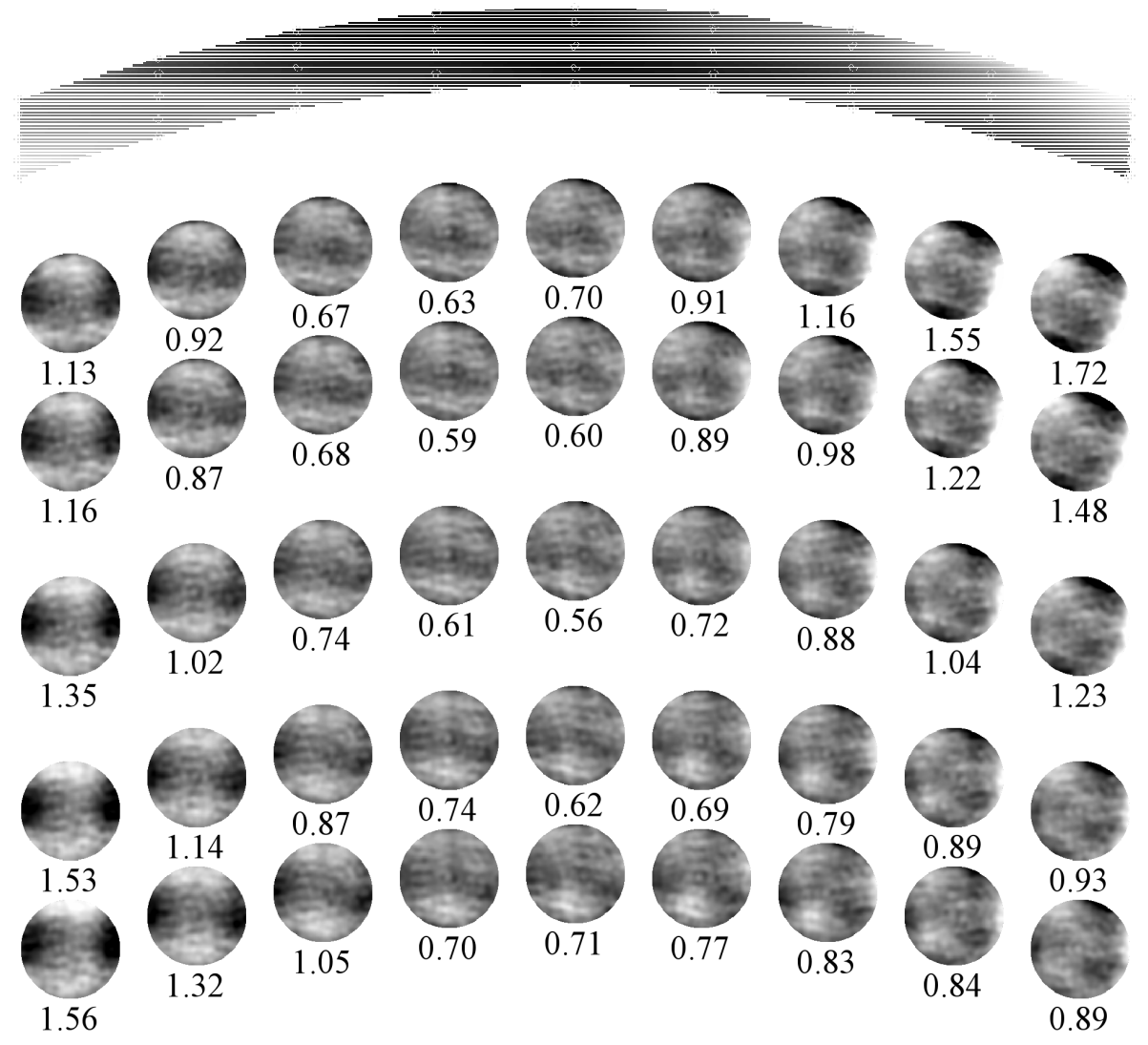

Fig 2 Wavefronts measured at each of the 45 different field points and contour map of the rms error across the field. The rms wavefront errors listed below each wavefront are in $\mathrm{nm}$ and are based on a 37-term Zernike polynomial fit to the wavefront with the measurementdependent piston, tilt, and focus terms removed. The depicted wavefronts include higher spatial frequency content than is contained within the 37-term Zernike polynomial reconstructions. Each wavefront image is individually scaled. 
Printed at full width of 19picas, do not reduce.

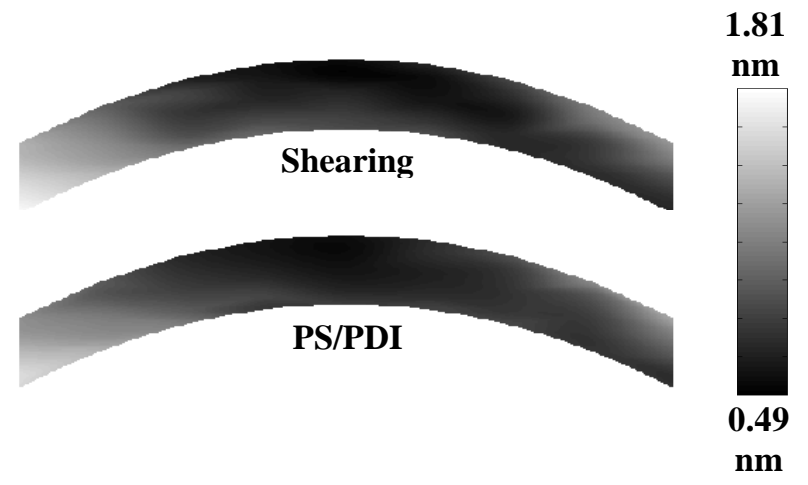

Fig. 3. Comparison between the final shearing measurement and the final PS/PDI measurement (both at-wavelenth). The contour maps are based on the rms error over a numerical aperture (NA) of 0.0915 as limited by the measurement NA of the shearing implementation used. For the comparison, the PS/PDI data was re-analyzed over the same grid size and NA as used for the shearing. The average agreement across the field is $(0.25 \pm 0.06) \mathrm{nm}$. 
Printed at full width of

35 picas, do not reduce.

Wavelength (nm)

13.10

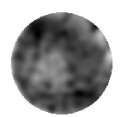

0.017

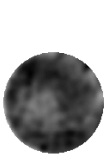

0.013

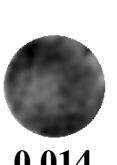

0.014

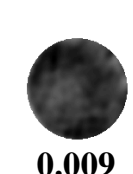

0.009

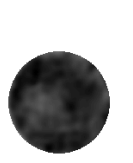

0.008
13.35

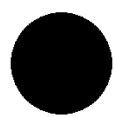

0.000

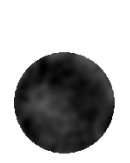

0.007

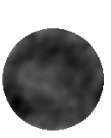

0.007

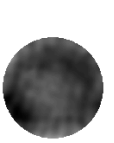

0.012

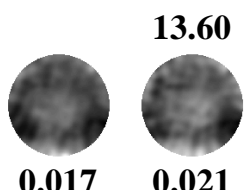

$0.017 \quad 0.021$

Difference wavefront rms magnitude (nm)

$0.00 \mathrm{~nm}$ 0.16 nm

Fig. 4. Wavefront variation, as a function of wavelength, relative to the wavefront measured at a wavelength of $13.35 \mathrm{~nm}$. This measurement was performed at the central field point where the wavefront error is approximately $0.6 \mathrm{~nm}$ rms. 
Printed at full width of 19picas, do not reduce.

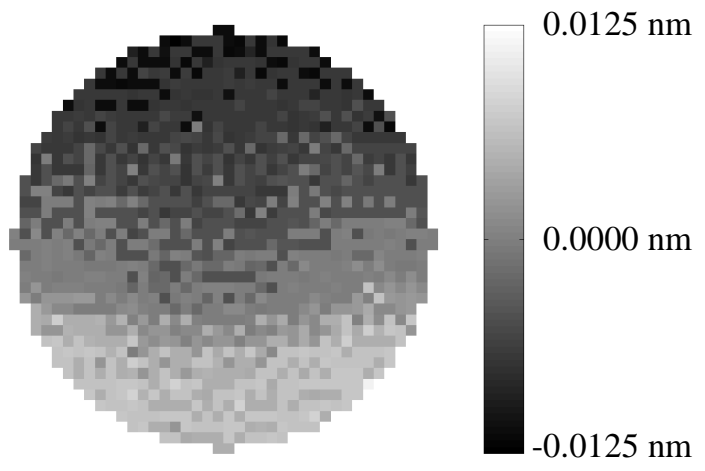

Fig. 5. Centroid wavelength change as a function of pupil position as measured at the central field point. The average centroid wavelength is $13.35 \mathrm{~nm}$ with a peak-to-valley linear variation of $(0.015 \pm 0.002) \mathrm{nm}$ across the pupil. Modeling results show an expected linear change of approximately $0.017 \mathrm{~nm}$ across the pupil. 